\title{
Note \\ Linear Stability of Finite Difference Equations for Three-Dimensional Flow Problems
}

For stable simulations of fluid flows by explicit finite difference schemes, the time step must be smaller than a critical time step. This critical time step can be predicted in general for linearized equations only. The technique for evaluating this time step is described in many textbooks, e.g., [1,2]. Nevertheless, often the rigorous analysis has been replaced by some rule of thumb, especially for problems in more than one space dimension and for multi-time-level methods with both convective and diffusive transports. In these cases the critical time step can no longer be given in explicit algebraic form. Therefore, several authors have assumed that the critical time step is equal to the minimum value found in some special limiting cases, such as pure convection or pure diffusion. In this respect the DuFortFrankel scheme $[1,2]$ seems to be advantageous, as it is unconditionally stable for pure diffusive transport. A combination of this scheme and the leapfrog scheme [1,2] for the convective terms has been proposed, therefore, by many authors [2-6]. The purpose of this note is to show that the critical time step is generally smaller than the minimum value found for pure convection and pure diffusion, especially for the combined leapfrog and DuFort-Frankel schemes.

We consider the following linearized finite difference equations.

Scheme I, leapfrog and DuFort-Frankel:

$$
\left[\left(v_{i, j, k}^{n+1}-v_{i, j, k}^{n-1}\right) / 2 \Delta t\right]+K_{a}{ }^{n}=D_{a}{ }^{n},
$$

Scheme II, leapfrog and time-lagged Euler:

$$
\left[\left(v_{i, j, k}^{n+1}-v_{i, j, k}^{n-1}\right) / 2 \Delta t\right]+K_{a}^{n}=D_{b}^{n-1},
$$

Scheme III, "upwind" differences and Euler:

$$
\left[\left(v_{i, j, k}^{n+1}-v_{i, j, k}^{n}\right) / \Delta t\right]+K_{b}{ }^{n}=D_{b}{ }^{n},
$$

where

$$
\begin{aligned}
K_{a}{ }^{n}= & (U / 2 \Delta x)\left(v_{i+1, j, k}^{n}-v_{i-1, j, k}^{n}\right)+(V / 2 \Delta y)\left(v_{i, j+1, k}^{n}-v_{i, j-1, k}^{n}\right) \\
& +(W / 2 \Delta z)\left(v_{i, j, k+1}^{n}-v_{i, j, k-1}^{n}\right),
\end{aligned}
$$




$$
\begin{aligned}
K_{b}{ }^{n}= & {[(U+|U|) / 2 \Delta x]\left(v_{i, j, k}^{n}-v_{i-1, j, k}^{n}\right)+[(U-|U|) / 2 \Delta x]\left(v_{i+1, j, k}^{n}-v_{i, j, k}^{n}\right) } \\
& +[(V+|V|) / 2 \Delta y]\left(v_{i, j, k}^{n}-v_{i, j-1, k}^{n}\right)+[(V-|V|) / 2 \Delta y]\left(v_{i, j+1, k}^{n}-v_{i, j, k}^{n}\right) \\
& +[(W+|W|) / 2 \Delta z]\left(v_{i, j, k}^{n}-v_{i, j, k-1}^{n}\right)+[(W-|W|) / 2 \Delta z]\left(v_{i, j, k+1}^{n}-v_{i, j, k}^{n}\right), \\
D_{a}{ }^{n}= & \mu\left\{\left(1 / \Delta x^{2}\right)\left[v_{i+1, j, k}^{n}-\left(v_{i, j, k}^{n+1}+v_{i, j, k}^{n-1}\right)+v_{i-1, j, k}^{n}\right]\right. \\
& +\left(1 / \Delta y^{2}\right)\left[v_{i, j+1, k}^{n}-\left(v_{i, j, k}^{n+1}+v_{i, j, k}^{n-1}\right)+v_{i, j-1, k}^{n}\right] \\
& \left.+\left(1 / \Delta z^{2}\right)\left[v_{i, j, k+1}^{n}-\left(v_{i, j, k}^{n+1}+v_{i, j, k}^{n-1}\right)+v_{i, j, k-1}^{n}\right]\right\}, \\
& \\
D_{b}{ }^{n}= & \mu\left\{\left(1 / \Delta x^{2}\right)\left[v_{i+1, j, k}^{n}-2 v_{i, j, k}^{n}+v_{i-1, j, k}^{n}\right]+\left(1 / \Delta y^{2}\right)\left[v_{i, j+1, k}^{n}-2 v_{i, j, k}^{n}+v_{i, j-1, k}^{n}\right]\right. \\
& \left.+\left(1 / \Delta z^{2}\right)\left[v_{i, j, k+1}^{n}-2 v_{i, j, k}^{n}+v_{i, j, k-1}^{n}\right]\right\} .
\end{aligned}
$$

Here, $K$ and $D$ can be interpreted as the convective and diffusive terms, respectively; $v$ is the dependent quantity, e.g., velocity. $U, V, W$ are the convective velocities; $\Delta x, \Delta y, \Delta z$ are the space increments of the Cartesian finite difference grid; $\mu$ is the viscosity; and $\Delta t$ is the time step. The subscripts $i, j, k$ label the grid points in space; the superscript indicates the time level. In order to allow the linear analysis $U, V, W, \mu$ must be constants and any volume or pressure forces have been neglected.

The general solution for these equations is

$$
v_{i, j, k}=A_{k_{1}, k_{2}, k_{3}}^{n} \prod_{j=1}^{3}\left(C_{j}+i S_{j}\right)
$$

where $i \equiv \sqrt{-1}$

$$
\begin{gathered}
C_{j}=\cos \left(k_{j} \Delta x_{j}\right), \quad\left(\Delta x_{1}, \Delta x_{2}, \Delta x_{3}\right)=(\Delta x, \Delta y, \Delta z), \\
S_{j}=\sin \left(k_{j} \Delta x_{j}\right), \quad j=1,2,3
\end{gathered}
$$

and $k_{1}, k_{2}, k_{3}$ are any wavenumbers. The variation of the amplitude $A_{k_{1}, k_{2}, k_{3}}^{n}$ vs time step $n$ is governed by the amplification matrix $\mathbf{G}$ ([1, Section 5]; for algebraic details, see [7]):

$$
\mathbf{G}=\left\{\begin{array}{cc}
2(a+i b) & c \\
1 & 0
\end{array}\right\}
$$

Here, the real parameters $a, b, c$ depend upon the scheme used. 
Scheme I:

$$
\begin{aligned}
& a=\frac{2 \mu \Delta t}{(1+\gamma)}\left[\frac{C_{1}}{\Delta x^{2}}+\frac{C_{2}}{\Delta y^{2}}+\frac{C_{3}}{\Delta z^{2}}\right], \\
& b=\frac{-\Delta t}{(1+\gamma)}\left[\frac{U S_{1}}{\Delta x}+\frac{V S_{2}}{\Delta y}+\frac{W S_{3}}{\Delta z}\right], \\
& c=(1-\gamma) /(1+\gamma), \\
& \gamma=2 \mu \Delta t\left[\frac{1}{\Delta x^{2}}+\frac{1}{\Delta y^{2}}+\frac{1}{\Delta z^{2}}\right] .
\end{aligned}
$$

Scheme II:

$$
\begin{aligned}
& a=0 \\
& b=-\Delta t\left[\frac{U S_{1}}{\Delta x}+\frac{V S_{2}}{\Delta y}+\frac{W S_{3}}{\Delta z}\right] \\
& c=1+4 \mu \Delta t\left[\frac{C_{1}-1}{\Delta x^{2}}+\frac{C_{2}-1}{\Delta y^{2}}+\frac{C_{3}-1}{\Delta z^{2}}\right] .
\end{aligned}
$$

Scheme III:

$$
\begin{aligned}
a= & \Delta t\left[\left(C_{1}-1\right)\left(\frac{|U|}{\Delta x}+\frac{2 \mu}{\Delta x^{2}}\right)+\left(C_{2}-1\right)\left(\frac{|V|}{\Delta y}+\frac{2 \mu}{\Delta y^{2}}\right)\right. \\
& \left.+\left(C_{3}-1\right)\left(\frac{|W|}{\Delta z}+\frac{2 \mu}{\Delta z^{2}}\right)\right], \\
b= & -\Delta t\left[\frac{U S_{1}}{\Delta x}+\frac{V S_{2}}{\Delta y}+\frac{W S_{3}}{\Delta z}\right], \\
c= & 0 .
\end{aligned}
$$

The critical time step $\Delta t$ follows from the Neumann criterion $[1,2]$, which requires for stability that neither of the two eigenvalues of $\mathbf{G}$ exceed the value one in magnitude. If we assume continuously distributed wavenumbers, then this criterion must be satisfied for all values of $C_{j}$ and $S_{j}$ with $C_{j}{ }^{2}+S_{j}{ }^{2}=1$.

This critical time step can be given algebraically for Scheme III:

$$
\Delta t \leqslant\left[\frac{|U|}{\Delta x}+\frac{|V|}{\Delta y}+\frac{|W|}{\Delta z}+2 \mu\left(\frac{1}{\Delta x^{2}}+\frac{1}{\Delta y^{2}}+\frac{1}{\Delta z^{2}}\right)\right]^{-1},
$$

which can be deduced from the analysis of Krause [8], too. We see here that $\Delta t$ must be smaller than those values found for either $U=V=W=0$ or $\mu=0$. 
This result can be explained geometrically. For this purpose let us assume $\Delta x=\Delta y=\Delta z$ and $U=V=W$. Then in two space dimensions, the usual five-point stencil can be enclosed in a rectangle the sides of which are separated by the distance $(2 / \sqrt{2}) \Delta x$ instead of $2 \Delta x$ as in the one-dimensional case. Also, the two velocity components result in a total of $\sqrt{2} U$. In three dimensions the seven-point stencil can be enclosed in an octahedron in which the smallest distance between two parallel planes is $(2 / \sqrt{3}) \Delta x$ and the resultant velocity is $\sqrt{3} U$. With these projected spacings and velocities we get the same results as (4) from the one-dimensional analysis. The viscous and convective terms add together as the total transport velocity is, e.g., $U+2 \mu / \Delta x$.

For the other schemes the critical time step can be given in explicit form for these limiting cases only:

(i) Zero convection (pure diffusion), $U=V=W=0$ :

Scheme I: $\Delta t \geqslant 0$, no restriction;

Scheme II: $\Delta t \leqslant \Delta t_{D} \equiv \frac{1}{4 \mu}\left[\frac{1}{\Delta x^{2}}+\frac{1}{\Delta y^{2}}+\frac{1}{\Delta z^{2}}\right]^{-1}$.

(ii) Zero diffusion (pure convection), $\mu=0$ :

Schemes I and II: $\Delta t \leqslant \Delta t_{C} \equiv\left[\frac{|U|}{\Delta x}+\frac{|V|}{\Delta y}+\frac{|W|}{\Delta z}\right]^{-1}$.

For the general case of combined diffusion and convection $\Delta t$ must be determined numerically. Some results for a special example,

$$
U=30, \quad V=3, \quad W=3, \quad \Delta x=0.125, \quad \Delta y=0.05, \Delta z=0.05
$$

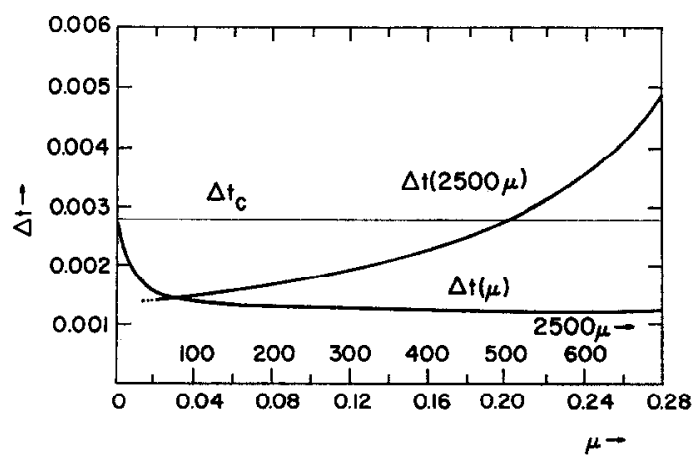

Fig. 1. Example for the critical time step $\Delta t$ for small $\left(\mu<\mu_{C}\right)$ and large $\left(\mu \gg \mu_{C}\right)$ values of the viscosity $\mu$ for Scheme I (leapfrog and DuFort-Frankel). $\Delta t_{C}$ is the limit for pure convection. 
(in arbitrary units), are shown in Fig. 1 for Scheme I. We see that $\Delta t=\Delta t_{C}$ is not permitted unless the viscosity $\mu$ is either zero or much larger than a viscosity $\mu_{C}$ characterizing the convective transport:

$$
\mu_{C}=\left(\frac{|U|}{\Delta x}+\frac{|V|}{\Delta y}+\frac{|W|}{\Delta z}\right)\left(\frac{1}{\Delta x^{2}}+\frac{1}{\Delta y^{2}}+\frac{1}{\Delta z^{2}}\right)^{-1} .
$$

In this example $\mu_{C} \approx 0.4$ and we see that $\mu$ must be larger than about $1250 \mu_{C}$ in order to permit $\Delta t=\Delta t_{C}$. For small viscosities the critical time step is less than $50 \%$ of $\Delta t_{C}$ in this (and similar) examples. (Fromm [3] used a "security factor" 0.5 in calculating $\Delta t$ from $\Delta t_{C}$.)

This pathological behavior of the combined leapfrog and DuFort-Frankel schemes does not exist in its one-dimensional form. Here, $\Delta t=\Delta t_{C}$ is permitted for any value $\mu \geqslant 0$ the viscosity $[2,3]$. It does exist already, however, in the two-dimensional case [7].

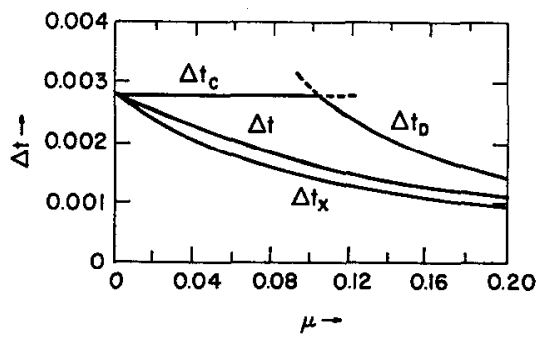

Fig. 2. Example for the critical time step $\Delta t$ vs viscosity $\mu$ for Scheme II (leapfrog and lagged Euler). $\Delta t_{C}=$ limit for pure convection; $\Delta t_{D}=$ limit for pure diffusion; $\Delta t_{X}=$ lower boundary.

For Scheme II, the critical time step $\Delta t$ is shown for this example in Fig. 2 as a function of the viscosity $\mu$. Again, we find that $\Delta t$ must be smaller than the minimum of $\Delta t_{C}$ and $\Delta t_{D}$ for $\mu>0$. However, for small values of the viscosity, $\Delta t$ can be larger than that value permitted for Scheme I. Without proof we notice that

$$
\Delta t_{X}=\left[\frac{|U|}{\Delta x}+\frac{|V|}{\Delta y}+\frac{|W|}{\Delta z}+4 \mu\left(\frac{1}{\Delta x^{2}}+\frac{1}{\Delta y^{2}}+\frac{1}{\Delta z^{2}}\right)\right]^{-1}
$$

seems to be an acceptable lower boundary for the critical time step in this case.

Scheme II together with Eq. (10) (reduced by a factor of 0.5 in order to account for nonlinearities) has been used successfully to simulate time-dependent and three-dimensional high Reynolds number channel flows [7]. 


\section{REFERENCES}

1. R. D. Richtmyer AND K. W. Morton, "Difference Methods for Initial-Value Problems," 2nd ed., Interscience, New York/London, 1967.

2. P. J. RoACHE, "Computational Fluid Dynamics," Hermosa, Albuquerque, 1972.

3. J. Fromm, Methods Comp. Phys. 3 (1964), 345.

4. Y. Rimon, Ph.D. Thesis, Princeton University, 1968.

5. D. G. Fox, J. Atmos. Sci. 29 (1972), 322.

6. D. G. Fox AND J. W. DeARdoRfF, J. Heat Transfer C94 (1972), 337.

7. U. SchumanN, Ein Verfahren zur direkten numerischen Simulation turbulenter Strömungen in Platten- und Ringspaltkanälen und über seine Anwendung zur Untersuchung von Turbulenzmodellen, Dissertation, Universität Karlsruhe, Report KFK 1854 (1973).

8. E. KRAUSE, Z. Angew. Math. Mech. 51 (1971), T 156.

RECEIVED: February 20, 1975

U. SCHUMANN*

Institut für Reaktorentwicklung Kernforschungszentrum Karlsruhe Karlsruhe, West Germany

* On leave (1974-1975) with the Advanced Study Program of the National Center for Atmospheric Research, Boulder, Colorado, which is sponsored by the National Science Foundation. 